\title{
Self-Injurious Thoughts and Behaviors Interview-Korean Version: Psychometric Properties
}

\author{
Seo Jeong Lee ${ }^{1}$, Ye Jin $\mathrm{Cho}^{2}$, and Myoung Ho Hyun ${ }^{2 凶}$ \\ 1Department of Psychiatry, Chung-Ang University Hospital, Seoul, Republic of Korea \\ ${ }^{2}$ Department of Psychology, Chung-Ang University, Seoul, Republic of Korea
}

Objective There is currently no structured interview tool developed that comprehensively evaluates self-injurious thoughts and behaviors (SITB) in Korea. The Self-injurious Thoughts and Behaviors Interview (SITBI) collectively measures suicidal ideation, plans, gestures, attempts, and non-suicidal self-injuries (NSSI). The SITBI's reliability and validity have been established with it being widely used in English speaking countries. This study evaluated the psychometric validity of the Korean version of the SITBI (SITBI-K).

Methods The SITBI's validity as a diagnostic assessment tool for NSSI and suicidal behavior disorder (SBD), as defined by the Diagnostic and Statistical Manual of Mental Disorders-5th edition, was examined. Analyses were performed on 108 university students reporting experiences of suicidal thoughts and behaviors (female 84.26\%, mean age $=22.10, \pm$ SD 3.33).

Results The SITBI-K displayed excellent interrater reliability, with a credible test-retest reliability at two months. Construct validity examined the correlation between the SITBI-K's modules and approved the self-report results. Appropriate convergent and discriminant validities were obtained for suicidal ideation, plans, gestures, attempts, and NSSI.

Conclusion The SITBI-K showed excellent psychometric validity at a level comparable to the original. Its clinical utility for both NSSI and SBD diagnoses was confirmed.

Psychiatry Investig 2021;18(2):157-165

Key Words SITBI-K, Non-suicidal self-injury, Suicidal behavior disorder.

\section{INTRODUCTION}

The Diagnostic and Statistical Manual of Mental Disorders5th edition (DSM-5) includes suicidal ideation and attempts as cross-cutting symptom measures that are recommended in identifying and diagnosing patients with mental health problems. ${ }^{1}$ Additionally, diagnoses for suicidal behavior disorder (SBD) and non-suicidal self-injury (NSSI) are presented as recommended topics for future studies to encourage interest among both clinicians and systematic researchers. This reflects the fact that self-injury related problems have become intensified on a global scale.

The suicide rate of Korea in 2018 was 24.7 per 100,000 peo-

Received: August 13, 2020 Revised: October 29, 2020

Accepted: December 5, 2020

$\triangle$ Correspondence: Myoung Ho Hyun, PhD

Department of Psychology, Chung-Ang University, 84 Heukseok-ro, Dongjak-gu, Seoul 06974, Republic of Korea

Tel: +82-2-820-5125, Fax: +82-2-816-5124, E-mail: hyunmh@cau.ac.kr

(c) This is an Open Access article distributed under the terms of the Creative Commons Attribution Non-Commercial License (https://creativecommons.org/licenses/by$\mathrm{nc} / 4.0$ ) which permits unrestricted non-commercial use, distribution, and reproduction in any medium, provided the original work is properly cited. ple, ranking it first among the Organization for Economic Cooperation and Development (OECD) countries. Initially, this rate was declining as of 2010, but is now showing an increase. ${ }^{2}$ According to the children and adolescents mental health survey conducted by the Ministry of Health and Welfare on middle and high school students across four areas, 17\% reported suicidal ideations, $3.7 \%$ reported behaviors with suicidal intentions, and 5.8\% reported committing self-injurious behaviors without suicidal intentions. ${ }^{3}$ Furthermore, according to the national Adolescent Mental-Health and Problem-Behavior Screening survey, conducted by the Ministry of Education on middle and high school students in 2018, 7.9\% of middle school and $6.4 \%$ of high school students reported engaging in selfinjurious behaviors without suicidal intentions. ${ }^{4}$ Finally, in a study conducted on psychiatric outpatients diagnosed with depressive symptoms, $42 \%$ reported engaging in suicide attempts, with $54 \%$ reporting history of NSSI. ${ }^{5}$

These issues can be divided into suicides with intention and self-injurious behaviors without suicidal intentions. Additionally, suicidal ideation, plans, and attempts are all suicide-related concepts, whereas suicidal gestures and NSSI thoughts are 
related to self-injurious behaviors without suicidal intentions. ${ }^{6}$ Additionally, the presence of suicidal plans indicates an increased risk of future suicide attempts. ${ }^{7}$ Furthermore, NSSI is associated with suicide, ${ }^{8}$ and has been discussed as a strong longitudinal predictor of future suicidal attempts. ${ }^{9}$ About $70 \%$ of adolescents reporting NSSI also engaged in suicide attempts, with clear suicidal intentions. ${ }^{10,11}$ Regardless of suicidal intentions and their severity, self-injurious behaviors are an important predictive factor that can lead to one committing suicide. ${ }^{12,13}$ Therefore, with regards to the research and clinical approach in this area, it is important for each self-injurious thoughts and behaviors (SITB) concept to be evaluated individually, based on clear operational definitions, in addition to the identifying of their relevance and influence on one another.

A factor hindering research on suicide-related behaviors is the lack of appropriate assessment tools. Although basic outlines of operational definitions for each SITB exist, most tools do not employ consistent definitions and sometimes measure only restricted ranges, causing limitations in the interpretations and comparisons of research conclusions based on them. ${ }^{14}$ In other words, it is difficult to thoroughly identify the relationship between behaviors using such tools due to their measurement restrictions. In addition, individual tools used in previous studies vary, causing limitations in comparing them.

Prior to the development of the SITBI, a tool that comprehensively measures suicide-related behaviors was developed. The Suicide Attempt Self-Injury Interview (SASII) is a structured 31-item measurement tool. ${ }^{15}$ The SASII distinguishes the experiences of the interviewee as either parasuicide, serious or non-serious suicide attempts, or NSSI. However, the SASII does not include suicidal ideation, plans, and self-injurious thoughts; while it allows for a simple assessment using only a few items, it produces limited relevant information. In addition, the Columbia-Suicide Severity Rating Scale (C-SSPS) is a clinical measure of suicide risk. ${ }^{16}$ It has the advantage of being able to divide suicidal behavior into severity of suicidal thoughts, strength of suicidal thoughts, suicidal behaviors, and lethality of suicidal behavior. In addition, it can be used to provide comprehensive measures. However, it does not include suicidal gestures and self-injurious ideation and behaviors as well as provide information as comprehensive as the SITBI. As such, there are various scales measuring each concept of SITBs, of which some are comprehensively measured. However, there are no tools to measure self-injury-related behaviors in an omnibus format that provide a rich background information like the SITBI-K.

Nock et al. ${ }^{14}$ developed and validated a structured interview that measures SITBs in an omnibus format. Venta and Sharp ${ }^{17}$ extended its concurrent validity on adolescent inpatients, with Spanish and German versions then being published as well. ${ }^{18,19}$
The SITBI is a structured interview tool conducted by a clinician, which includes a long-form version of 169 items, and a short-form version of 72 items. Interviews consist of five modules: suicidal ideation, plans, gestures, attempts, and NSSI. Each module begins with a screening question that checks for lifetime presence and, if none has occurred, subsequent items are skipped in order to move onto the next module. If no SITB are present, the interview ends after six screening questions (NSSI is divided into thoughts and behaviors) across the five modules. If a lifetime presence is reported, subsequent items specifically question frequency (frequency during lifetime, past year, past month, and past week), age of onset, most recent occurrence of SITB and specific methods utilized, severity of the behavior, precipitants and functions, level of physical pain during suicide attempts, proportion of alcohol and drug use, periods of thought before engaging in the behavior, numbers and level of influences from peers, and future likelihood of SITB.

The number of papers citing Nock et al.'s ${ }^{14}$ SITBI development study comes to 518 (ProQuest search on June 1st, 2020), with researches using the SITBI on multiple samples of varying backgrounds recently accumulating. ${ }^{20,21}$ There are currently no assessment tools that comprehensively assess SITBs based on specific and clear conceptual definitions, in Korea. The development of such tools will help to apply realistic clinical characteristics into SITB research, allowing for the development of a multidimensional approach. ${ }^{14}$ Along with introducing various self-injury related assessment tool, the need to develop a measure suited to the Korean context has become clear. Additionally, the need to secure the reliability and validity of common tools that are widely used at home and abroad has been raised by researchers. ${ }^{22}$ As such, this study aimed to develop a Korean version of the SITBI and to assess its reliability, validity and applicability in clinical diagnoses.

\section{METHODS}

\section{Participants}

From March to July 2019, research participation advertisements were made available both online and offline in three universities located in Seoul, Korea. Informed written consent forms were obtained from each of the volunteers. The selection criteria included adults aged 18 years or older and who had, reported one or more SITB. The exclusion criteria based on research ethics, included "those reporting self-injuries requiring medical treatment within the last month" and "those who had received inpatient treatment due to specific mental disorders within the last month." Those unable to make reliable selfreports, such as people with formal thought disorder, intellectual disability, and organic mental disorder were also excluded. Other exclusion criteria included those who were not fluent 
in Korean, and those who had their last SITB experience more than 10 years prior to this study.

\section{Measures}

\section{The Korean Version of Self-Injurious Thoughts and} Behaviors (SITBI-K) ${ }^{14}$

The long-form version of this tool, consisting of 169 items was used, which took around 5-30 minutes. First, the six screening questions asking on the lifetime presence of each SITB were answered as either yes or no (e.g." "Have you ever had thoughts of killing yourself?"). Then, the subsequent 97 items asking on the severity of each SITB experience or the degree of influence of preceding and related factors were presented as a five point Likert scale (e.g., "On a scale of 0 to 4 , when you engaged in NSSI, how much did you do it as a way to get rid of bad feelings?"). The other 66 items asking for the presence, specific methods and reasons for the SITBs etc., were presented as open-ended questions (e.g., "Why do you think you engage in NSSI behaviors?").

\section{The Scale for Suicidal Ideation (SSI $\left.{ }^{23}\right)$}

The SSI contains 19 items that are rated using a 3-point Likert scale ranging from $0-2$. It is used to measure the existence of suicidal ideation, attitudes about suicide, and severity of suicidal plan, as well as to confirm the convergent validity of suicidal ideation and attempts in the SITBI-K. The self-report version of this measure, as converted by Shin et al., ${ }^{24}$ was used. Its internal consistency $(\alpha)$ in this study was 0.85 .

\section{Suicidal Intent Scale (SIS ${ }^{25}$ )}

The SIS consists of 15 items that are rated using a 3-point Likert scale ranging from $0-2$. It is used to assess the severity of suicidal intentions of suicide attempters as well as to check the convergent validity of suicidal plans and attempts in the SITBI-K, which are based on suicidal intentions. In addition, it determines discriminant validity using suicidal gestures and self-injury. We used the translated self-report version ${ }^{26}$ of the SIS in this study.

\section{Beck Depression Scale-II-Item 9 'Suicidal Thoughts or Wishes' (BDI-II ${ }^{27}$ )}

The BDI-II is comprised of a 4-point Likert scale. A study on psychiatric outpatients reported that scoring two or more points on this scale indicates that the probability of them attempting suicide is 6.9 times higher than average. ${ }^{28}$

\section{Survival and Coping Beliefs of the Reason for Living $\left(\mathrm{RFL}^{29}\right)$}

The RFL was developed to assess potential reasons for not committing suicide. We used the translated version of this tool $^{30}$ in our study. As Linehan et al. ${ }^{29}$ suggested utilizing the scores by factor rather than the total RFL score, the "survival and coping beliefs" subscale was used. We used participants' total score in the subscale to check for discriminant validity of suicidal ideation and attempts. The internal consistency $(\alpha)$ of the subscale in the current research was 0.95 .

\section{The Functional Assessment of Self-Mutilation (FASM ${ }^{31}$ )}

To measure the methods and frequency of self-injurious behaviors, a version of this scale, as translated by Kwon and Kwon, ${ }^{32}$ was used. A total score from 12 items in Group A, asking after participants' self-injurious methods and frequency, was calculated.

\section{Procedure}

This study's procedure was approved by the University Institutional Review Board and complied with the Declaration of Helsinki (IRB No: 1041078-201801-HRSB-019-01K). The scale translation procedure adhered to the International Test Commission guidelines. ${ }^{33}$ All interviews were conducted in accordance with the guidelines provided by Nock et al., ${ }^{14}$ wherein three people, including two licensed clinical psychologists and one $\mathrm{PhD}$ in clinical psychology, conducted the interviews after prior training and practical sessions. Participants completed the self-report questionnaires for about 15 minutes after the interview and returned home after receiving a remuneration. There were no occurrences of emergency situations or complaints about inconveniences during the research process.

\section{Data analysis}

Of the 120 people interviewed, data from five participants were excluded: one participant with psychosis was excluded due to unreliability in reporting, while the other four (aged 32 years or older) were excluded because their data hindered the homogeneity of the group due to their relatively older ages. Upon conducting frequency analysis, seven anomalous values that dramatically broadened the frequency of the SITB were excluded as well, resulting in a finalized total of 108 participants. For reference, 97 local residents and 111 inpatients were included as participants to develop and establish the reliability and validity of the original scale ${ }^{14}$ and the German version of SITBI, ${ }^{19}$ respectively. Meanwhile, 106 inpatients were included in the study extending its concurrent validity. ${ }^{17}$

The SITBI is not a psychometric tool that measures a single concept using multiple items of single format, but an interview tool that uses various formats of items to measure a wide range of constructs. Therefore, factor analysis and internal consistency reliability coefficients are not calculated together, and such methods do not always hold a theoretical or technical 
meaning. ${ }^{14}$ Instead, we carefully conducted a descriptive statistics analysis (mean, standard deviation, ratio, and range) using the reported responses in the current sample, and compared it with the prior study ${ }^{14,17,19}$ to confirm the appropriateness of the construct. Furthermore, construct validity was confirmed by checking whether frequency of SITBs, which is a key item of the SITBI, converged or discriminated with the conceptually similar and validity-confirmed original scale.

IBM SPSS Statistics version 23 (IBM Corp., Armonk, NY, USA) was used for the statistical analysis. Descriptive analyses were conducted on participants' demographic and SITB data. Convergent and discriminant validities between the frequency of SITB in the SITBI-K and the validated self-report scale scores were checked. Interrater reliability, test-retest reliability, and concordance of diagnoses were calculated to ensure this measure's applicability for diagnostic uses.

\section{RESULTS}

\section{SITBI-K descriptive statistics}

In total, 91 out of 108 interviewees were female (84.26\%), with the sample's mean age being 22.10 years ( \pm SD 3.33; ranging from 18 to 31 years). Descriptive statistics of the SITBI-K are shown in Table 1. Lifetime presence of SITBs showed that 10 participants (98.1\%) reported suicidal ideation, 74 (68.5\%) reported suicidal plans, 18 (16.7\%) reported suicidal gestures, 47 (43.5\%) reported suicide attempts, and 68 (63.0\%) reported NSSI. Further examination of the suicide attempts and NSSI revealed that three participants $(2.8 \%)$ attempted suicide and $11(10.2 \%)$ experienced NSSI within the last week. Lifetime frequency of the SITBs was 3.2 ( \pm SD 2.99) times higher than average for suicide attempts, 93.93 ( \pm SD 198.30) times higher for NSSI; within the last week, these frequencies were 0.04 $( \pm$ SD 0.21$)$ times for suicide attempts, and 0.24 ( \pm SD 0.60$)$ times higher for NSSI.

\section{Interrater reliability}

Five cases were randomly selected from the recording files for the raters to listen to in order for them to assess and compare. Likert scale items of the lifetime presence, frequency, severity, reinforcement, and precipitant factors of the SITB were obtained. The presence of each module was analyzed using Cohen's Kappa ( $\kappa)$, with the frequency being analyzed using a binary correlation coefficient. Results close to 0 indicate a disagreement between the raters, with those close to 1 indicating an agreement. ${ }^{34}$ The lifetime presence, and frequency, as well as the frequency during the past year, past month, and past week for the five modules, all showed perfect consistency (all ks=1, rs=1). These results are identical to those of Nock et al.'s ${ }^{14}$ study, possibly due to the obtaining of the presence of
SITB and quantifiable responses using structured questions.

\section{Test-retest reliability}

In total, 20 participants from the first interview were selected for the short-form interview (72 items), which was conducted after an interval of about two months. During the first interview, all participants were informed regarding the reassessment required for the reliability study; most voluntarily agreed to participate and were sent a reminder message regarding such, wherein they were asked for their available date and time for a phone interview. Participants with an easily adjustable interview schedule were prioritized for selection. Subsequent interviews reported presence of SITB, frequency, and other related variables during the period from when the first interview was conducted to the second one. The presence of each module was analyzed using $\kappa$, with lifetime frequency and frequency during the last year being analyzed using a one-way random effects Intraclass Correlation Coefficient (ICC). ${ }^{35}$

The results for the consistency of the presence of SITB showed that suicidal ideation and plans possessed a perfect consistency of $\kappa=1$, with suicide attempts and self-injurious behaviors showing moderate consistencies of $\kappa=0.48$ and $\kappa=0.42$, respectively. Conversely, suicidal gesture showed a relatively low consistency of $\kappa=0.33$. The results for consistency of the lifetime frequency of SITB indicated a perfect consistency (ICC $=0.91$, $\mathrm{p}<0.000)$ for suicidal plans and intermediate consistencies (ICC $=0.72, \mathrm{p}<0.010$ and $\mathrm{ICC}=0.48, \mathrm{p}<0.018$, respectively) for suicide attempts and NSSI. The consistency results for suicidal ideation were not significant, but did indicate a trend (ICC= $0.67, \mathrm{p}<0.054)$; with those for suicidal gestures not being significant at all $(\mathrm{ICC}=0.30, \mathrm{p}<0.250)$. Similar patterns of results were found for the consistency of these frequencies in the past year.

\section{Construct validity}

Analyses were conducted based on the frequencies during the "past year" and "past month," wherein retrospective reporting bias is expected to be relatively small, yet sufficient responses can still be obtained. The correlation coefficient results of the frequency of each SITBI-K module and the construct validity scales are presented in Table 2.

Frequencies of suicidal ideations, plans, gestures, and attempts showed moderate or larger correlations, with the total scores for the SSI suicidal ideation and BDI-II Item 9 suicidal thought severity indicating convergence $(\mathrm{r}=0.338-0.680, \mathrm{p}<$ $0.01 ; \mathrm{r}=0.304-0.561, \mathrm{p}<0.05)$. Furthermore, the correlation between frequency for each module of the SITBI-K and the suicidal intent section of the SIS total scores were examined. While suicidal plans and attempts showed low to moderate correlations with the SIS ( $r=0.405-0.479, \mathrm{p}<0.01 ; \mathrm{r}=0.328-0.336, \mathrm{p}<$ 
Table 1. Descriptive statistics for the SITBI-K (N=108)

\begin{tabular}{|c|c|c|c|c|c|}
\hline & Suicidal ideation & Suicidal plan & Suicidal gesture & Suicidal attempt & NSSI \\
\hline & $\mathrm{N}(\%)$ & $\mathrm{N}(\%)$ & $\mathrm{N}(\%)$ & $\mathrm{N}(\%)$ & $\mathrm{N}(\%)$ \\
\hline \multicolumn{6}{|l|}{ Presence } \\
\hline Lifetime & $106(98.1)$ & $74(68.5)$ & $18(16.7)$ & $47(43.5)$ & $68(63.0)$ \\
\hline Past year & $86(79.6)$ & $36(33.3)$ & $7(6.4)$ & $24(22.2)$ & $47(63.6)$ \\
\hline Past month & $54(50.0)$ & $15(13.9)$ & $5(4.6)$ & $6(5.5)$ & $22(20.4)$ \\
\hline \multirow[t]{2}{*}{ Past week } & $37(43.2)$ & $6(5.6)$ & $2(1.9)$ & $3(2.8)$ & $11(10.2)$ \\
\hline & $\mathrm{M} \pm \mathrm{SD}$ & $\mathrm{M} \pm \mathrm{SD}$ & $\mathrm{M} \pm \mathrm{SD}$ & $\mathrm{M} \pm \mathrm{SD}$ & $\mathrm{M} \pm \mathrm{SD}$ \\
\hline \multicolumn{6}{|l|}{ Frequency } \\
\hline Lifetime & $417.59 \pm 730.10$ & $22.26 \pm 72.30$ & $9.53 \pm 14.20$ & $3.20 \pm 2.99$ & $93.93 \pm 198.30$ \\
\hline Past year & $67.75 \pm 127.79$ & $6.75 \pm 20.47$ & $1.18 \pm 1.63$ & $0.84 \pm 1.21$ & $11.81 \pm 22.32$ \\
\hline Past month & $4.93 \pm 9.57$ & $0.77 \pm 2.88$ & $0.35 \pm 0.61$ & $0.09 \pm 0.29$ & $1.43 \pm 3.49$ \\
\hline Past week & $1.42 \pm 3.37$ & $0.15 \pm 0.68$ & $0.12 \pm 0.33$ & $0.04 \pm 0.21$ & $0.24 \pm 0.60$ \\
\hline Age of onset & $14.77 \pm 4.44$ & $16.78 \pm 4.85$ & $16.88 \pm 3.53$ & $17.26 \pm 4.59$ & $16.48 \pm 3.45$ \\
\hline \multicolumn{6}{|l|}{ Severity $(1-4)$} \\
\hline Worst point & $3.68 \pm 0.57$ & $3.62 \pm 0.59$ & - & - & - \\
\hline Average & $2.34 \pm 0.77$ & $2.53 \pm 0.93$ & - & - & - \\
\hline \multicolumn{6}{|l|}{ Reinforcement (1-4) } \\
\hline Automatic negative & $2.45 \pm 1.33$ & $2.20 \pm 1.45$ & $1.71 \pm 1.31$ & $2.19 \pm 1.47$ & $3.06 \pm 1.01$ \\
\hline Automatic positive & $1.04 \pm 1.32$ & $0.81 \pm 1.09$ & $0.76 \pm 1.30$ & $0.96 \pm 1.41$ & $1.44 \pm 1.51$ \\
\hline Social negative & $1.00 \pm 1.23$ & $0.88 \pm 1.19$ & $3.00 \pm 1.37$ & $1.00 \pm 1.18$ & $1.00 \pm 1.23$ \\
\hline Social positive & $3.10 \pm 1.00$ & $3.28 \pm 0.88$ & $2.12 \pm 1.32$ & $3.13 \pm 1.17$ & $2.22 \pm 1.45$ \\
\hline \multicolumn{6}{|l|}{ Precipitant (1-4) } \\
\hline Family & $2.49 \pm 1.41$ & $2.42 \pm 1.46$ & $2.82 \pm 1.56$ & $2.66 \pm 1.36$ & $2.37 \pm 1.36$ \\
\hline Friends & $1.46 \pm 1.34$ & $1.11 \pm 1.36$ & $0.94 \pm 1.35$ & $1.21 \pm 1.40$ & $1.19 \pm 1.32$ \\
\hline Relationship & $1.68 \pm 1.37$ & $1.66 \pm 1.44$ & $1.41 \pm 1.46$ & $1.62 \pm 1.45$ & $1.29 \pm 1.34$ \\
\hline Peers & $1.40 \pm 1.42$ & $1.34 \pm 1.45$ & $1.06 \pm 1.39$ & $1.21 \pm 1.40$ & $1.00 \pm 1.28$ \\
\hline Work/school & $2.08 \pm 1.36$ & $1.85 \pm 1.55$ & $1.35 \pm 1.27$ & $1.68 \pm 1.66$ & $1.84 \pm 1.46$ \\
\hline Mental state & $3.38 \pm 0.80$ & $3.49 \pm 0.67$ & $2.65 \pm 1.54$ & $3.70 \pm 0.51$ & $3.26 \pm 0.92$ \\
\hline \multicolumn{6}{|l|}{ Characteristics } \\
\hline Physical pain (1-4) & - & - & - & $1.87 \pm 1.44$ & - \\
\hline Alcohol/drug use (\%) & $20.75 \pm 32.02$ & $20.55 \pm 34.38$ & $13.00 \pm 27.98$ & $21.70 \pm 37.32$ & $11.62 \pm 24.01$ \\
\hline Peers with behavior before 1st time (No.) & $1.03 \pm 2.00$ & $0.83 \pm 2.09$ & $0.50 \pm 1.10$ & $0.91 \pm 1.87$ & $1.03 \pm 3.50$ \\
\hline Peers with behavior after 1st time (No.) & $2.92 \pm 6.45$ & $1.22 \pm 3.44$ & $0.53 \pm 0.87$ & $0.78 \pm 1.83$ & $1.97 \pm 4.35$ \\
\hline Peer influence before 1 st time (1-4) & $0.50 \pm 1.07$ & $0.53 \pm 1.10$ & $0.47 \pm 1.18$ & $0.66 \pm 1.19$ & $0.65 \pm 1.22$ \\
\hline Peer influence after 1st time (1-4) & $0.54 \pm 0.97$ & $0.39 \pm 0.79$ & $0.41 \pm 1.00$ & $0.26 \pm 0.71$ & $0.53 \pm 1.00$ \\
\hline \multicolumn{6}{|l|}{ Period of thought before engage in (\%) } \\
\hline $0 \mathrm{sec}$ & 0.0 & 0.0 & 2.8 & 1.9 & 11.1 \\
\hline $1-60 s$ & 1.9 & 1.9 & 4.6 & 0.9 & 13.9 \\
\hline $2-15 \min$ & 0.0 & 2.8 & 3.7 & 1.9 & 17.6 \\
\hline $16-60 \mathrm{~min}$ & 11.1 & 12.0 & 2.8 & 6.5 & 9.3 \\
\hline Less 1 day & 29.6 & 12.0 & 1.9 & 7.4 & 4.6 \\
\hline 1-2 days & 12.0 & 9.3 & 0.0 & 6.5 & 2.8 \\
\hline More 2 days & 28.7 & 24.1 & 0.0 & 15.7 & 0.9 \\
\hline Wide range & 14.8 & 6.6 & 0.0 & 2.8 & 2.8 \\
\hline Future likelihood of this behavior (1-4) & $2.66 \pm 1.15$ & $2.01 \pm 1.36$ & $1.29 \pm 1.40$ & $1.91 \pm 1.32$ & $1.9 \pm 1.43$ \\
\hline
\end{tabular}

SITBI-K: Korean version of the Self-injurious Thoughts and Behaviors Interview 
Table 2. Spearman correlation between continuous SITBI-K variables and concurrent validity measures

\begin{tabular}{|c|c|c|c|c|c|}
\hline & $\begin{array}{l}\text { Suicidal Ideation } \\
\text { (SSI total) }\end{array}$ & $\begin{array}{l}\text { Suicidal Intention } \\
\quad \text { (SIS total) }\end{array}$ & $\begin{array}{l}\text { Suicidal Ideation } \\
\text { Intensity } \\
\text { (BDI-II item 9) }\end{array}$ & $\begin{array}{c}\text { Self-Injury } \\
\text { (FASM item A total) }\end{array}$ & $\begin{array}{c}\text { RFL } \\
\text { survival \& coping } \\
\text { beliefs total }\end{array}$ \\
\hline & $\mathrm{M} \pm \mathrm{SD}$ & $\mathrm{M} \pm \mathrm{SD}$ & $\mathrm{M} \pm \mathrm{SD}$ & $\mathrm{M} \pm \mathrm{SD}$ & $\mathrm{M} \pm \mathrm{SD}$ \\
\hline SITBI-K & $17.75 \pm 6.62$ & $15.71 \pm 4.21$ & $1.18 \pm 0.84$ & $18.75 \pm 12.04$ & $55.91 \pm 17.93$ \\
\hline \multicolumn{6}{|c|}{ Suicidal ideation } \\
\hline Past year & $0.436^{\dagger}$ & 0.142 & $0.338^{\dagger}$ & 0.121 & $-0.321^{\dagger}$ \\
\hline Past month & $0.509^{\dagger}$ & $0.304^{*}$ & $0.502^{\dagger}$ & 0.073 & $-0.442^{\dagger}$ \\
\hline \multicolumn{6}{|l|}{ Suicidal plan } \\
\hline Past year & $0.481^{\dagger}$ & $0.336^{*}$ & $0.372^{\dagger}$ & -0.049 & $-0.382^{\dagger}$ \\
\hline Past month & $0.561^{\dagger}$ & $0.405^{\dagger}$ & $0.539^{\dagger}$ & -0.058 & $-0.462^{\dagger}$ \\
\hline \multicolumn{6}{|l|}{ Suicidal gesture } \\
\hline Past year & $0.680^{\dagger}$ & 0.132 & $0.616^{\dagger}$ & $0.674^{*}$ & -0.465 \\
\hline Past month & $0.561^{*}$ & -0.354 & $0.671^{\dagger}$ & 0.434 & $-0.542^{*}$ \\
\hline \multicolumn{6}{|l|}{ Suicidal attempt } \\
\hline Past year & $0.521^{\dagger}$ & $0.479^{\dagger}$ & $0.516^{\dagger}$ & 0.147 & $-0.469^{\dagger}$ \\
\hline Past month & $0.451^{\dagger}$ & $0.328^{*}$ & $0.497^{\dagger}$ & 0.212 & $-0.445^{\dagger}$ \\
\hline \multicolumn{6}{|l|}{ NSSI } \\
\hline Past year & $0.331^{\dagger}$ & -0.009 & $0.363^{\dagger}$ & $0.380^{\dagger}$ & $-0.324^{\dagger}$ \\
\hline Past month & $0.281^{*}$ & -0.077 & $0.395^{\dagger}$ & $0.259^{*}$ & $-0.299^{*}$ \\
\hline
\end{tabular}

${ }^{*} \mathrm{p}<0.05,{ }^{\dagger} \mathrm{p}<0.01$. SITBI-K: Korean version of the Self-injurious Thoughts and Behaviors Interview, SSI: The Scale for Suicide Ideation, SIS: Suicidal Intent Scale, BDI-II: Beck Depression Inventory-II, FASM: The Functional Assessment of Self-Mutilation, RFL: Survival and Coping Beliefs of the Reason for Living

0.05), with suicidal attempts indicating convergence, correlations between suicidal gestures and NSSI were not significant and were thus discriminated ( $\mathrm{r}=-0.009--0.354, \mathrm{~ns})$. These results are in line with the definition of suicidal gestures, which are actions that are intended to make others believe in one's own suicide attempt "without the intention of dying" as well as the definition of NSSI, which are specified as self-injuries "without suicidal intentions." Total scores of the RFL's Survival and Coping Beliefs for Reason of Living section showed an inverse correlation with the suicidal ideation, plans, and attempts sections of the SITBI-K ( $\mathrm{r}=0.321-0.469, \mathrm{p}<0.01)$. Additionally, NSSI frequency within the SITBI-K showed convergence as it is moderately correlated with FASM's Group A items ( $\mathrm{r}=$ $-0.380, \mathrm{p}<0.01 ; \mathrm{r}=-0.259, \mathrm{p}<0.05)$, and was discriminated as it did not show significant correlation with suicidal ideation, plans, or attempts ( $r=-0.049-0.434$, ns). Thus, the construct validity of the SITBI-K has been established.

\section{Diagnostic applicability}

For the randomly selected group of 52 participants, a clinical psychologist, with 14 years of career experience, diagnosed both NSSI and SBD based on the DSM-5 criteria. The SITBI$\mathrm{K}$ items conducted by another interviewer were structured for diagnostic use, and were also checked for their diagnostic con- sistency. The diagnostic structure form of the SITBI-K is presented in Supplementary Table 1 (in the online-only Data Supplement), which is a partially edited version of that used in the German study. ${ }^{19}$ Note that the NSSI diagnostic criteria E ("distress or interference in important areas of functioning") and $\mathrm{F}$ ("differentiation between other mental disorders or medical conditions"), as well as the SBD diagnostic criterion D ("differentiation between states of delirium or confusion") were not identifiable through using the SITBI-K, and were thus excluded for calculating consistency.

The results indicate that the SBD diagnosis showed a perfect consistency $(\kappa=1.0)$, with NSSI showing an excellent consistency $(\kappa=0.94)$. For the NSSI diagnosis, 2 of the 52 cases showed inconsistencies for diagnostic criteria B ("functions of self-injury"). This resulted from the experts' strict application of criterion $\mathrm{B}$, rather than using the subjective responses of the interviewees.

\section{DISCUSSION}

SITB includes suicidal ideation, plans, gestures, attempts, and NSSI. In clinical research and interventions, discriminative yet comprehensive assessments based on clear definitions of each of these variables are needed for effective diagnoses 
and treatment. Only then can research reflect the actual, real life occurrences of these phenomena, which will then help researchers and clinicians to understand the relationships and influences between each of these variables. Furthermore, this will allow clinicians to consider multiple aspects of a patient's self-injurious behaviors in the development of effective interventions. This study translated a structured interview tool (SITBI), that uniformly assesses SITBs into Korean. Additionally, its reliability, validity, and clinical utility as a diagnostic assessment measure were examined.

The descriptive results of the SITBI-K analysis were similar to those of Nock et al. $\mathrm{s}^{14}$ original study, as well as those of the German study by Fischer et al. ${ }^{19}$ However, the frequencies of suicidal ideation and NSSI were largely different in this study. The frequency of NSSI in this study was similar to that of Fischer et al.'. ${ }^{19}$ German sample, but was only up to one-seventh of the results as reported in Nock et al.s ${ }^{14}$ United States (U.S.) sample. Furthermore, the frequency of suicidal ideation was five times higher than that of the U.S. sample. Overall, the results of the current study found suicidal ideation's frequency to be relatively high, with that of NSSI being relatively low. This may partially reflect the characteristics of the university sample, compared to the U.S. study that involved adolescents. However, it is also necessary to examine whether these results reflect Eastern cultural characteristics, wherein ideological-based activities are dominant compared to those involving acting out. The average ages of onset for the SITB were 14.77 years for suicidal ideation, and 16-17 years (M 16.48-17.26) for suicidal plans, gestures, attempts, and NSSI, which is a result comparable to those of a previous study. ${ }^{6}$

As for the reported functions of the SITB, (suicidal ideation, plans, attempts, and NSSI), there were relatively high numbers of responses stating they were used "as a way to get rid of bad feelings" (negative personal reinforcement) and "in order to get out of doing something or to get away from others" (negative social reinforcement). This is distinctive from the reasons given for suicidal gestures, wherein their most reported function was "in order to communicate with someone else or to get attention" (positive social reinforcement), a result consistent with its definition and the findings of previous studies. ${ }^{14,19}$ All SITBs reported two distinctly high levels of "the state of my mental health at the time" and "family problems," which were followed by "work/school problems" as their key precipitants. This is different from the U.S. and German studies which revealed "peer problems" to be the most common reason. This difference is considered to have been influenced by the fact that the interviewees had left adolescence, wherein peer pressure is magnified, as well as Korea's high university entrance examination stress.

The average number of participants that had exchanged in- fluences on SITBs was less than one. Additionally, most reported sharing either their peer's or their own experiences only after the occurrence and denied any direct influences on each other. This value is two to three times smaller than the U.S. and German samples. Conversely, there were numerous situations wherein the SITB of family members were reported on during the interviews. Family members displaying SITB may result in psychological trauma, as well as producing a learning effect, and can thus have more significant influences than those of one's peers. As there are no separate items to assess this matter, supplementation of the SITBI-K in considering the characteristics of Korean samples may be required in future.

As for the periods of thought before engaging in SITB, those lasting less than one day, and those lasting two or more days, showed the highest levels for suicidal ideation, with periods lasting two or more days occurring for suicidal plans and attempts, which is relatively long. Conversely, the periods of thought occurring before suicidal gestures generally lasted from one second to less than one day, with those occurring before NSSI lasting from zero to one second, which is relatively short. This reflects the characteristics of suicidal gestures and NSSI, wherein a relatively short period of thought is required when compared to other related behaviors, as well as acting out due to situational factors. The future likelihood of SITB showed mean scores of 1.29-2.66 ( $\pm \mathrm{SD} 1.15-1.43)$, but the number of those who reported four points for suicidal ideation, plans, attempts, and NSSI were 30 (27.8\%), 14 (13\%), 7 (6.5\%), and $14(13 \%)$, respectively.

Relatively stable interrater and test-retest reliabilities were confirmed for the SITBI-K. However, whether the test-retest reliability measures are appropriate for retrospective reporting still needs to be considered. Consistency for suicidal gestures was particularly low, a similar result to that of the previous study by Nock et al. ${ }^{14}$ Participants who reported suicidal gestures in the first interview did not respond in the follow-up interviews, thus, lowering the consistency. Therefore, there is a need to increase the sample size and reconfirm the finding. Moreover, this may be due to suicidal gestures-especially considering its conceptual characteristics-having a low number of responses, as well as its vulnerability to interference from subjective judgments relative to other behaviors. Additionally, convergent and discriminant validities of the five modules of the SITBI-K were obtained by comparing it against other existing and validated measurement tools.

The largest advantage of the SITBI-K is that it allows for the identification of the five types of SITB in an omnibus measure in a relatively short amount of time. Until now, this kind of tool was inexistent in Korea, which caused difficulties in organically studying various behaviors related to self-injury. It is the only tool that discriminates NSSI, suicide attempts, and 
suicidal gestures. Next, its clinical utility in diagnosing NSSI and SBD, as based on the DSM- 5 criteria, was demonstrated. However, its diagnostic accuracy may be raised further if clinicians can identify the presence of any NSSI reinforcement paradigms, problems functioning in important areas of daily life due to NSSI, as well as being able to establish differential diagnoses with other mental disorders. Finally, as the SITBI$\mathrm{K}$ has an advantage, in that it obtains an overall view of self-injurious behaviors in clinical situations within a short amount of time, it may be useful in identifying if suicidality, including suicidal plans and attempts, is also present in interviewees who report only the single problems of either self-injury or suicidal ideation. In addition, the SITBI-K may be useful in determining whether one self-injury-related behavior coexists or bidirectionally affects another self-injury-related behavior.

The sample in this study was comprised of students from universities in Seoul, representing a relatively highly educated example of all potential SITB samples. Therefore, generalization of the results of the current study may possess some limitations. It may be necessary to extend the validity through comparisons with samples of varying ages and backgrounds, such as adolescents (considering the average age of onset of SITBs) or inpatients. However, considering the frequency of suicide attempts and NSSI, and the future likelihood of self-injurious behaviors, a significant proportion of the current sample belongs in the subclinical group. Furthermore, a study examining patients who have reported self-injurious behaviors, who were admitted to the emergency rooms of 20 Korean university hospitals in the past five years, showed that people from the ages of 20-29 years constituted the largest proportion. In addition, considering that the prevalence rate of 16-year old adolescents with SITB is similar to that of adults aged 20-29 years, ${ }^{36}$ a sample comprised of participants aged 18 years or older, specifically those in their early 20s, may be an appropriate choice for studies focusing on people with a Korean background. Moreover, additional research can be conducted by jointly using self-injury-related structured interview tools such as the SASII ${ }^{15}$ and SITBI-K to secure the concurrent validity. It would also be ideal to supplement the criterion validity through longitudinally monitoring suicide attempts and occurrence of NSSI, among others, during the process of extending this tool's validity. Finally, the SITBI-K contains open-ended questions that ask specific methods or reasons for SITBs, which were not included in the validation process through quantitative analysis. As such, subsequent studies may examine reasons and methods of suicidal attempts or self-injuries as well as situational or environmental factors that contribute to SITBIs through qualitative analysis.

\section{Supplementary Materials}

The online-only Data Supplement is available with this article at https://doi.org/10.30773/pi.2020.0302.

\section{Acknowledgments}

This research was supported by the Chung-Ang University Excellent Student Scholarship in 2020.

\section{Conflicts of Interest}

The authors have no potential conflicts of interest to disclose.

\section{Author Contributions}

Conceptualization: Seo Jeong Lee, Myung Ho Hyun. Data curation: Seo Jeong Lee, Ye Jin Cho. Formal analysis: Seo Jeong Lee. Investigation: Seo Jeong Lee, Ye Jin Cho. Methodology: Seo Jeong Lee, Myung Ho Hyun. Software: Seo Jeong Lee. Validation: Seo Jeong Lee. Writing-original draft: Seo Jeong Lee. Writing_review \& editing: Myung Ho Hyun, Ye Jin Cho.

\section{ORCID iDs}

Seo Jeong Lee

Ye Jin Cho

https://orcid.org/0000-0002-0359-3943

\section{REFERENCES}

1. American Psychiatric Association. Diagnostic and Statistical Manual of Mental Disorders (DSM- $\left.5^{\circledR}\right)$. Washington DC: American Psychiatric Pub; 2013.

2. Statistics Korea. Cause of death statistics. Available at: http://www.index.go.kr/unify/idx-info.do?idxCd=8040. Accessed March 24, 2020.

3. Jung YS. $17.6 \%$ of middle and high school students think suicide. Medical News. Available at: http://www.bosa.co.kr/news/articleView. html?idxno=2088603. Accessed March 25, 2020.

4. Lee JH, Cho YY, Jeon JY. 70,000 middle and high school students' selfharm experience: Results from Ministry of Education's Adolescent Mental-Health and Problem - Behavior Screening Questionnaire. Hankyoreh21. Available at: http://www.hani.co.kr/arti/society general/869668. html. Accessed March 30, 2020.

5. Kim C, Lee S, Chang H. Influence of non-suicidal self-injury on suicide attempt among depressed patients. Korean J Clin Psychol 2018; 37:465-478.

6. Nock MK, Favazza AR. Non-Suicidal Self-Injury: Definition and Classification. In: Nock MK, Editor. Understanding Nonsuicidal Self-Injury: Origines, Assessment, and Treatment. Washington, DC: American Psychological Association, 2009, p.9-18.

7. Kessler RC, Borges G, Walters EE. Prevalence of and risk factors for lifetime suicide attempts in the National Comorbidity Survey. Arch Gen Psychiatry 1999;56:617-626.

8. Joiner TE, Ribeiro JD, Silva C. Nonsuicidal self-injury, suicidal behavior, and their co-occurrence as viewed through the lens of the interpersonal theory of suicide. Curr Dir Psychol Sci 2012;21:342-347.

9. Wilkinson P, Kelvin R, Roberts C, Dubicka B, Goodyer I. Clinical and psychosocial predictors of suicide attempts and nonsuicidal self-injury in the Adolescent Depression Antidepressants and Psychotherapy Trial (ADAPT). Am J Psychiatry 2011;168:495-501.

10. Nock MK, Joiner Jr TE, Gordon KH, Lloyd-Richardson E, Prinstein MJ. Non-suicidal self-injury among adolescents: diagnostic correlates and relation to suicide attempts. J Psychiatr Res 2006;144:65-72.

11. Nock MK. Self-injury. Annu Rev Clin Psychol 2010;6:339-363.

12. Brent D. Practicioner review: the aftercare of adolescents with deliberate selfharm. J Child Psychol Psychiatry 1997;38:277-286.

13. Olfson M, Gameroff MJ, Marcus SC, Greenberg T, Shaffer D. Emergency treatment of young people following deliberate self-harm. Arch 
Gen Psychiatry 2005;62:1122-1128.

14. Nock MK, Holmberg EB, Photos VI, Michel BD. Self-Injurious thoughts and behaviors interview: development, reliability, and validity in an adolescent sample. Psychol Assess 2007;19:309-317.

15. Linehan MM, Comtois KA, Brown MZ, Heard HL, Wagner A. Suicide Attempt Self-Injury Interview (SASII): development, reliability, and validity of a scale to assess suicide attempts and intentional self-injury. Psychol Assess 2006;18:303-312.

16. Posner K, Brent D, Lucas C, Gould M, Stanley B, Brown G, et al. Columbia-Suicide Severity Rating Scale (C-SSRS). New York, NY: Columbia University Medical Center; 2008.

17. Venta A, Sharp C. Extending the concurrent validity of the Self-Injurious Thoughts and Behaviors Interview to inpatient adolescents. J Psychopathol Behav Assess 2014;36:675-682.

18. García-Nieto R, Blasco-Fontecilla H, Yepes MP, Baca-García E. Traducción y validación de la Self-Injurious Thoughts and Behaviors Interview en población española con conducta suicida. Rev Psiquiatr Salud Ment 2013;6:101-108.

19. Fischer G, Ameis N, Parzer P, Plener PL, Groschwitz R, Vonderlin E, et al. The German version of the self-injurious thoughts and behaviors interview (SITBI-G): a tool to assess non-suicidal self-injury and suicidal behavior disorder. BMC Psychiatry 2014;14:265-273.

20. Klonsky E. Non-suicidal self-injury in United States adults: prevalence, sociodemographics, topography and functions. Psychol Med 2011;41: 1981-1986.

21. Boffa JW, Stanley IH, Hom MA, Norr AM, Joiner TE, Schmidt NB. PTSD symptoms and suicidalthoughts and behaviors among firefighters. J Psychiatr Res 2017;84:277-283.

22. Seong Y, Bae Y, Kim S. Non-suicidal self-injury in South Korea: a systematic review of studies from 2000 to 2018. Cogn Behav Ther Kor 2019;19:251-280.

23. Beck AT, Kovacs M, Weissman A. Assessment of suicidal intention: the Scale for Suicide Ideation. J Consult Clin Psychol 1979;47:343-352.
24. Shin MS, Park KB, Oh KJ, Kim ZS. A study of suicidal ideation among high school students: the structural relation among depression, hopelessness, and suicidal ideation. Korean J Clin Psychol 1990;9:1-19.

25. Beck AT, Weissman A, Lester D, Trexler L. Classification of suicidal behaviors: II. Dimensions of suicidal intent. Arch Gen Psychiatry 1976; 33:835-837.

26. Linehan MM. Suicide Intent Scale: Self-Report Form. Unpublished inventory. Seattle, Washington: University of Washington; 1982.

27. Beck AT, Ward CH, Mendelson M, Mock J, Erbaugh J. An inventory for measuring depression. Arch Gen Psychiatry 1961;4:561-571.

28. Brown GK. A Review of Suicide Assessment Measures for Intervention Research with Adults and Older Adults. Washington D.C.: USA National Institute of Mental Health; 2001.

29. Linehan MM, Goodstein JL, Nielsen SL, Chiles JA. Reasons for staying alive when you are thinking of killing yourself: the Reasons for Living Inventory. J Consult Clin Psychol 1983;51:276-286.

30. Lee G, Hyun M, Lee S. A study on the validation of the reasons for living inventory. Korean J Health Psychol 2010;15:227-242.

31. Lloyd-Richardson EE, Perrine N, Dierker L, Kelley ML. Characteristics and functions of non-suicidal self-injury in a community sample of adolescents. Psychol Med 2007;37:1183-1192.

32. Kwon H, Kwon S. Validation study of the Korean version of the Functional Assessment of Self-Mutilation (FASM). Clin Psychol Kor Res Pract 2017;3:187-205.

33. International Test Commission. ITC guidelines for translating and adapting tests (Second edition). Int J Test 2018;18:101-134.

34. Fleiss JL, Levin B, Paik MC. Statistical Methods for Rates and Proportions. New York: John Wiley \& Sons; 2013.

35. Shrout PE, Fleiss JL. Intraclass correlations: uses in assessing rater reliability. Psychol Bull 1979;86:420-428.

36. Jung KY, Kim T, Hwang SY, Lee TR, Yoon H, Shin TG, et al. Deliberate self-harm among young people begins to increase at the very early age: a nationwide study. J Korean Med Sci 2018;33:1-12. 
Supplementary Table 1. Operationalization of the diagnoses NSSI and SBD proposed in the DSM-5 by using the SITBI-K

$$
\text { DSM-5 Non-Suicidal Self-Injury }
$$

A. In the last year, the individual has, on 5 or more days, engaged in intentional self-inflicted damage to the surface of his or her body of a sort likely to induce bleeding, bruising, or pain (e.g., cutting, burning, stabbing, hitting, excessive rubbing), with the expectation that the injury will lead to only minor or moderate physical harm (i.e., there is no suicidal intent)

B. The individual engages in the self-injurious behavior with one or more of the following expectations:

1. To obtain relief from a negative feeling or cognitive state

2. To resolve an interpersonal difficulty

3. To induce a positive feeling state

C. The intentional self-injury is associated with at least one of the following: 1. Interpersonal difficulties or negative feelings or thoughts, such as depression, anxiety, tension, anger, generalized distress, or self-criticism, occurring in the period immediately prior to the self-injurious act

2. Prior to engaging in the act, a period of preoccupation with the intended behavior that is difficult to control

3. Thinking about self-injury that occurs frequently, even when it is not acted upon

D. The behavior is not socially sanctioned (e.g., body piercing, tattooing, part of religious or cultural ritual) and is not restricted to picking a scab or nail biting

E. The behavior or its consequences cause clinically significant distress or interference in interpersonal, academic, or other important areas of functioning

F. The behavior does not occur exclusively during psychotic episodes, delirium, substance intoxication withdrawal. In individual with a neurodevelopmental disorder, the behavior is not part of a pattern of repetitive stereotypies. The behavior is not better explained by another medical disorder or medical condition (e.g., Psychotic dx., autism spectrum dx., intellectual disability, Lesch-Nyhan syn. stereotypic movement dx. with self-injury, trichotillomania, excoriation dx.)

$$
\text { DSM-5 Suicidal Behavior Disorder }
$$

A. With in the last 24 months, the individual has made a suicide attempt Specify if_Current: not more than 12 months since the last attempt. In early remission: 12-24 months since the last attempt

B. The act does not meet criteria for non-suicidal self-injury that is, it does not involve self-injury directed to the surface of the body undertaken to induce relief from a negative feeling/ cognitive state or to achieve a positive mood state

C. The diagnosis is not applied to suicidal ideation or to preparatory acts

D. The act was not initiated during a state of delirium or confusion

E. The act not undertaken solely for a political or religious objective
143) Have you ever actually engaged in NSSI?

147) How many times in the past year?

152) Why do you think you engage in NSSI?

153) On a scale of 0 to 4 , when you have engaged in NSSI, how much did you do it as a way to get rid of bad feelings?

154) How much did you engage in NSSI in order to feel something, because you were feeling numb or empty?

155) How much did you engage in NSSI to communicate with someone else or to get attention?

156) How much did you engage in NSSI to get out of doing something or to get away from others?

157) On a scale of 0 to 4 , to what extent did problems with your family lead to your engaging in NSSI?

158) How much did problems with your friends lead to your engaging in NSSI?

159) How much did problems with your relationships lead to your engaging in NSSI?

160) How much did problems with your peers lead to your engaging in NSSI?

161) How much did problems with work or school lead to your engaging in NSSI?

162) How much did your mental state at the time lead to your engaging in NSSI?

119) During how many separate times in your life have you thought about engaging in NSSI?

123) On the scale of 0 to 4 , at the worst point, how intense were your thoughts about engaging in NSSI?

124) On average, how intense were these thoughts?

137) When you have had these thoughts, how long have they usually lasted?

150) Now I'm going to go through a list of things that people have done to harm themselves. Please let me know which of these you've done:

1) cut or carved skin 2) hit yourself on purpose 3) pulled your hair out 4) gave yourself a tattoo 5) picked at a wound 6) burned your skin (i.e., with a cigarette, match or other hot object) 7) inserted objects under your nails or skin 8) bit yourself (e.g., your mouth or lip) 9) picked areas of your body to the point of drawing blood 10) scraped your skin 11) "erased" your skin to the point of drawing blood 12) other (specify): 88) not applicable 99) unknown

(Further screening)

(Pre-screening)

DSM-5: the Diagnostic and Statistical Manual of Mental Disorders-5th edition, SITBI-K: the Korean version of the Self-Injurious Thoughts and Behaviors Interview, NSSI: Non-Suicidal SelfInjury, SBD: Suicidal Behavior Disorder

(Differentiated form Suicidal ideation, Plan, \& Gesture)

(Pre-screening)

93) What were the circumstances that contributed most to your most recent attempt?

98) Why do you think you make suicide attempts?

84) Have you ever made an actual attempt to kill yourself in which you had at least some intent to die?

When was the most recent attempt?

89) How many have you made in the past year?

(Differentiated form NSSI) 